\title{
Declaración del Comité Consultivo de Inmunizaciones de la Sociedad Chilena de Infectología respecto a la vacuna anti- virus papiloma humano. Septiembre 2008
}

\author{
Katia Abarca V $\mathbf{1}$, M Teresa Valenzuela $\mathbf{B}^{\mathbf{2}}$, Rodrigo Vergara $\mathrm{F}^{\mathbf{3}}$, \\ Vivian Luchsinger $\mathrm{F}^{4}$, Alma Muñoz $\mathrm{M}^{5}$, Jorge Jiménez de la $\mathrm{J}^{6}$, \\ Erna Ripoll M7 , Miguel $\mathbf{O}^{\prime}$ Ryan $\mathbf{G}^{8}$. Comité Consultivo de \\ Inmunizaciones, Sociedad Chilena de Infectología.
}

\section{Human papillomavirus vaccine. Statement of the Advisory Committee of Immunizations on Behalf of the Chilean Infectious Diseases Society. September 2008}

This article briefly reviews the epidemiology of human papillomavirus (HPV) infection and associated diseases globally and in Chile, and the scientific information of the licensed HPV vaccines: Gardasil ${ }^{\circledR}$ and Cervarix ${ }^{\circledR}$. Considering the available information, the Advisory Committee on Immunizations of the Chilean Society of Infectious Diseases recommends vaccination of teenage girls, ideally before initiating sexual activity, i.e. approximately at the age of 12 to 13 years and vaccination of women of any age if they have not started sexual activity. If women are vaccinated after initiating sexual activity, they should be informed of the lower efficacy of immunization if HPV infection has occurred. Education on responsible sexuality and sexually transmitted diseases should be maintained as a priority. Vaccination should be highly considered for inclusion in the National Immunization Program (Rev Méd Chile 2008; 136: 1485-92).

(Key words: Human papilloma virus; Oncogenic viruses; Uterine cervical neoplasms)

\begin{abstract}
Recibido el 16 de septiembre, 2008. Aceptado el 6 de octubre, 2008.
${ }_{1}^{1}$ Departamento de Pediatría, Facultad de Medicina, Pontificia Universidad Católica de Chile. ${ }^{2}$ Departamento de Salud Pública y Epidemiología, Facultad de Medicina, Universidad de Los Andes. ${ }^{3}$ Departamento de Preclínicas y Pediatría, Escuela de Medicina, Universidad de Valparaíso y Servicio de Pediatría, Hospital Carlos van Buren, Valparaíso. ${ }^{4}$ Programa de Virología, Instituto de Ciencias Biomédicas, Facultad de Medicina, Universidad de Chile. ${ }^{5}$ Centro para Vacunas en Desarrollo-Chile. ${ }^{6}$ Departamento de Salud Pública, Facultad de Medicina, Pontificia Universidad Católica de Chile. ${ }^{7}$ Servicio de Medicina, Hospital Carlos van Buren, Valparaíso. ${ }^{8}$ Programa de Microbiología y Micología, Instituto de Ciencias Biomédicas, Facultad de Medicina, Universidad de Chile.
\end{abstract}

Correspondencia a: Dra. Katia Abarca V. Centro de Investigaciones Médicas, Pontificia Universidad Católica de Chile. Marcoleta 391, $3^{\text {er }}$ piso, Santiago. Fonos: 354 6823, 354 6825. Fax: 638 7457. E mail: katia@med.puc.cl

Por acuerdo entre los autores y los Editores de la Revista Médica de Chile y la Revista Chilena de Infectología, este documento será publicado en ambas revistas. 


\section{Epidemiología mundial y regional del cáncer cérvico-uterino (CCU) y de la infección por virus papiloma humano:}

El CCU es el segundo cáncer en frecuencia entre las mujeres en el mundo, con cerca de medio millón de nuevos casos y 274.000 muertes cada año. La gran mayoría de los casos y las muertes ocurren en los países más pobres, donde no se han implementado sistemas eficaces de detección precoz ${ }^{1}$.

Desde hace más de una década, estudios epidemiológicos y moleculares han permitido demostrar causa-efecto entre la infección por virus papiloma humano (VPH) y el CCU. El rol del VPH como agente causal del CCU ha sido firmemente establecido, encontrándose ADN de este virus en $99,7 \%$ de muestras tumorales de mujeres de distintas zonas del mundo. Ocho tipos de VPH son responsables de $90 \%$ de los cánceres cervicales: 16, 18, 45, 31, 33, 52, 58 y 35. Los tipos 16 y 18 son los más comunes, dando cuenta de $70 \%$ de los cánceres de células escamosas y 89\% de los adenocarcinomas/carcinomas adenoescamosos, con leves variaciones entre las distintas regiones del mundo ${ }^{2}$.

En mujeres de América Latina y El Caribe se demostró una prevalencia de infección por VPH de $96,8 \%$ (IC 95\% 93,5-100) en 1.399 mujeres con lesiones precursoras de cáncer y de 88\% (IC 95\% 80,6-95,4) en 1.795 mujeres con CCU de tipo escamoso (Valenzuela MT, observaciones no publicadas).

Además de su participación claramente establecida en el CCU, los VPH de alto riesgo tienen participación en proporciones variables (entre $30 \%$ y $70 \%$ ) de otros cánceres, como los de vulva, vagina, ano, pene y orofaringe ${ }^{2-5}$. Los tipos de VPH 6 y 11 son los principales causantes de las verrugas genitales o condilomas acuminados y de la papilomatosis recurrente respiratoria ${ }^{6}$. Los datos regionales de estas patologías son escasos; un metaanálisis que recopila información de América Latina y El Caribe encontró una prevalencia de VPH de 81,5\% en 160 casos de condiloma acuminado (Valenzuela MT, observaciones no publicadas) y en $53 \%$ de 15 casos reportados de papilomatosis respiratoria recurrente, con presencia de los HPV 16, 6 y 117.

La infección genital por VPH se adquiere por contacto sexual; la mayor prevalencia en la vida ocurre en los primeros años de iniciada la actividad sexual, varía entre $20 \%$ y $40 \%$ entre los 15 y 25 años, luego baja progresivamente en mujeres de edad media para nuevamente mostrar un leve ascenso después de los 50-60 años (curva bimodal) ${ }^{3,4,8}$. En América Latina y países del Caribe la prevalencia de infección por VPH determinada mediante PCR en 21.600 mujeres sanas fue de 18,7\% (IC 95\% 15,3-22,0) (Valenzuela MT, observaciones no publicadas).

La infección genital por VPH suele ser autolimitada y aproximadamente $90 \%$ de las infecciones desaparecen en el curso de 2 años. Las infecciones que persisten por varios años se asocian a lesiones intraepiteliales, algunas de las cuales también pueden regresar. La persistencia prolongada de la infección lleva a una progresión clonal del epitelio persistentemente infectado pudiendo llegar a producir una neoplasia intraepitelial (NIE) grado 3, la que suele progresar a invasión cervical dando como resultado un CCU. Se estima que entre la infección y la neoplasia intraepitelial NIE grado 3 transcurren generalmente 7 a 15 años. Sin embargo, se han documentado casos de rápido desarrollo, incluso de unos pocos meses desde la infección ${ }^{9}$.

\section{Epidemiología en Chile:}

Carga de enfermedad. En nuestro país se presentan alrededor de 1.200 nuevos casos de CCU por año, con una tasa estimada de 28,8 por 100.000 mujeres mayores de 15 años beneficiarias del sistema público. El año 2004 ocurrieron 676 muertes por esta causa, constituyendo la quinta causa de muerte por cáncer en la mujer ${ }^{10}$.

Nuestro país cuenta con un programa de pesquisa y control del CCU desde hace más de 20 años. Una de sus principales actividades es el tamizaje citológico mediante Papanicolau periódico a mujeres entre 25 y 64 años de edad, alcanzando una cobertura de PAP vigente en los últimos 3 años en mujeres beneficiarias del Sistema Público en Chile de $68 \%$ en diciembre de 2007, cifra similar a la de los 5 años previos ${ }^{11}$. La mortalidad en Chile por CCU ha descendido de 14,3 a 8,5 por 100.000 en el período $1990-2003^{12}$.

Magnitud de la infección por virus papiloma humano. La prevalencia global de infección por VPH en muestras cervicales de cerca de 1.000 mujeres de 15 a 69 años de la Región Metropolitana fue de 
$14 \% 13$ y de $29 \%$ en más de 1.000 muestras cervicovaginales autocolectadas por mujeres sobre 17 años ${ }^{14}$. La prevalencia de infección más alta, cercana a $20 \%$, ocurre en mujeres menores de 20 años. Los VPH 16 y 18 se han encontrado en $26 \%$ a $34 \%$ de las mujeres estudiadas ${ }^{15}$.

El único estudio chileno publicado de VPH en muestras de CCU, detectó los tipos 16 o 18 en $47,3 \%$ de 19 muestras analizadas ${ }^{16}$. Dos estudios recientes, aún no publicados, en alrededor de 120 y 220 muestras de CCU, respectivamente, muestran una frecuencia de VPH 16 y 18 similar a lo descrito en el resto del mundo, esto es, cercanas a $70 \%$ (Suárez E, observaciones no publicadas; Valdivia I, et al. Libro de Resúmenes del XXXI Congreso Chileno de Obstetricia y Ginecología 2007; página 32).

No se dispone de datos epidemiológicos locales de la frecuencia de infección por VPH 6 y 11. En los centros centinelas de enfermedades de transmisión sexual se notificaron 427 casos de condiloma acuminado en el año 1999 (19,7\% del total de ETS notificadas ese año) ${ }^{17}, 2.309$ casos durante los años 1999 a 2003 (21,9\% del total de ETS notificadas en dicho período ${ }^{18}$ y cerca de la mitad de ellas en tres centros centinelas durante los años 2004 y $2005^{19}$.

Inicio de la actividad sexual en jóvenes chilenos. Encuestas realizadas en los años 2003 y 2004 muestran que un tercio de los jóvenes ha iniciado actividad sexual antes de los 15 años ${ }^{20}$ y que $7 \%$ a $12 \%$ de los varones y $1 \%$ a $3 \%$ de las mujeres han iniciado actividad sexual antes de los 13 años ${ }^{21}$.

\section{Vacunas contra VPH:}

Actualmente existen dos vacunas contra VPH, ambas licenciadas en nuestro país: Gardasil ${ }^{\circledR}$, vacuna cuadrivalente (VPH 6, 11, 16 y 18) de Merck Sharp \& Domme (MSD) y Cervarix ${ }^{\circledR}$, vacuna bivalente (VPH 16 y 18) de Glaxo SmithKline (GSK). Ambas están elaboradas con proteína L1ª en la forma de partículas similares a virus o VLPb.

- Gardasil $^{\circledR}$ : la proteína L1 es producida en células de levadura Saccharomyces cerevisae, contiene sulfato hidroxifosfato de aluminio amorfo como adyuvante y el esquema de vacunación es de tres dosis a 0,2 y 6 meses $^{22}$. - Cervarix ${ }^{\circledR}$ : la proteína L1 es producida en células de insecto (baculovirus), utiliza como adyuvante hidróxido de aluminio con un nuevo adyuvante AS04 (monofosforil lípido A deacilatado) ${ }^{23}$ y el esquema de vacunación es de tres dosis a 0,1 y 6 meses $^{24}$.

Inmunogenicidad. Los estudios realizados han mostrado que más de $99 \%$ de las mujeres entre 15 y 24 años y niñas y niños de 9 a 15 años responden a la vacunación con anticuerpos contra los 4 tipos de VPH contenidos en la vacuna. Los títulos de anticuerpos son mayores que con la infección natural, 2,7 veces mayores en niños y 1,7 veces mayores en niñas que en mujeres mayores $^{25-30}$. Estudios recientes en mujeres de 24 a 45 años muestran elevada seroconversión (98\%$100 \%)$, pero los niveles de anticuerpos son menores que los observados en mujeres más jóvenes (Luna J, et al. International Papillomavirus Congress Beijing, China 2007, Presentation \#PA1-04).

Los títulos de anticuerpos después de la tercera dosis van disminuyendo hasta el mes 24, alcanzan una meseta y se han mantenido estables durante los 60 meses de seguimiento a la fecha ${ }^{25,26,31-33}$.

Reactogenicidad y seguridad. Con ambas vacunas, entre $84 \%$ y $94 \%$ de los vacunados ha reportado dolor en el sitio de inyección y alrededor de 13\%$16 \%$ ha notificado fiebre, frecuencia similar a la que ocurre con placebo. La mayoría de las reacciones han sido leves y moderadas. Los eventos adversos sistémicos ocurrieron en baja frecuencia y en similar proporción de vacunados y receptores de placebo. La seguridad de la vacuna ha sido evaluada como satisfactoria, con muy escasos eventos adversos serios considerados relacionados con la vacuna (broncoespasmo, gastroenteritis, cefalea/hipertensión arterial, hemorragia vaginal, compromiso de la movilidad del brazo). No ha habido muertes relacionadas a la vacuna ${ }^{26,33-35}$. La

\footnotetext{
a Proteína L1, principal componente (80\%) de la cápsula viral de VPH y principal inmunógeno.

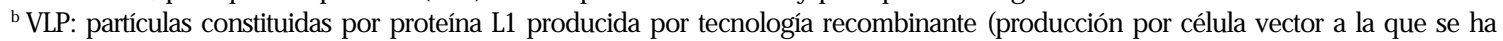
insertado el gen que codifica dicha proteína); en forma natural esta proteína se autoensambla en partículas con forma de vinus.
} 
vacunación en el embarazo ha mostrado similar frecuencia de abortos espontáneos y anormalidades congénitas en los grupos vacunados y placebo; su uso en embarazo se ha clasificado en categonía $\mathrm{B}^{\mathrm{c} 34,35 \text {. }}$

Evaluación de la eficacia de las vacunas anti VPH. Considerando que no es posible evaluar en forma directa la capacidad de las vacunas para prevenir el CCUd, las autoridades regulatorias han aceptado que la eficacia sea evaluada utilizando como metas sustitutas 0 alternativas los eventos previos al cáncer, como son las infecciones persistentes y las NIE de distinto grado. Del mismo modo, debido a la imposibilidad de evaluar estas metas en niñase, se acepta como información adecuada la obtenida en los llamados estudios "puente", esto es, al documentarse una buena inmunogenicidad en estos grupos etarios, se extrapola la eficacia demostrada en mujeres mayores.

Eficacia. Ambas vacunas han demostrado una excelente eficacia (cercana a 100\%) en evitar NIE grado 2 y 3 por virus 16 y 18 en pacientes que no estaban previamente infectadas. La Tabla 1 resume los resultados obtenidos en diversos estudios de eficacia de fase III con ambas vacunas.
Además, la vacuna cuadrivalente ha mostrado elevada eficacia para la prevención de verrugas genitales por tipos vacunales $(100 \%$, 95\% IC 94100); neoplasias vaginales y vulvares grado 2 ó 3 (100\%, 95\% IC 56-100); y lesiones genitales externas que incluyen neoplasia vaginal, vulvar y condilomas en mujeres latinoamericanas (100\%, 95\% IC 93,3-100) 25,30,31,37-39.

Eficacia en mujeres infectadas. En mujeres infectadas por los tipos de VPH contenidos en las vacunas, detectados mediante PCR en muestra de cérvix, la vacunación con vacuna cuadrivalente no mostró ningún efecto beneficioso ${ }^{25,31,39}$. Un estudio con vacuna bivalente en Costa Rica, en 2.189 mujeres de 18 a 25 años infectadas con VPH mostró que la vacunación no se asoció a eliminación viral a los 6 y 12 meses de seguimiento 40 . Estos datos indican que no existe evidencia de algún efecto terapéutico de la vacuna en mujeres ya infectadas por VPH vacunales.

Protección cruzada. Se ha establecido cierta protección contra otros genotipos virales relacionados con los incluidos en las vacunas y que pueden encontrarse asociados a CCU. A continuación se resume la información disponible respecto

Tabla 1.

\begin{tabular}{|c|c|c|c|c|}
\hline \multirow[t]{2}{*}{ M etas a evaluar } & \multicolumn{2}{|c|}{$\begin{array}{c}\text { Gardasi }^{\circledR} \\
\text { Seguimiento } 3 \text { años } \\
n=5.455 \text { y } n=12.167\end{array}$} & \multicolumn{2}{|c|}{$\begin{array}{c}\text { Cervarix }^{\circledR} \\
\text { Seguimiento } 15 \text { meses } \\
n=18.644\end{array}$} \\
\hline & $\begin{array}{l}\% \text { eficacia } \\
(95 \% \text { IC) }\end{array}$ & $\begin{array}{c}\text { Eventos } \\
\text { placebo/vacuna }\end{array}$ & $\begin{array}{l}\text { \% eficacia } \\
(95 \% \text { IC) }\end{array}$ & $\begin{array}{c}\text { Eventos } \\
\text { placebo/vacuna }\end{array}$ \\
\hline NIE 2 ó 3 por VPH 16/18 cohorte total & $95(85-99)$ & $62 / 3$ & $90(53-99)$ & $21 / 2$ \\
\hline NIE 2 ó 3 por VPH 16/18 población por protocolo & $98(86-100)$ & $42 / 1$ & $100(74-100)$ & $20 / 0$ \\
\hline NIE $1+$ por VPH 16 & $100(93-100)$ & $53 / 0$ & $94(54-100)$ & $17 / 1$ \\
\hline NIE $1+$ por VPH 18 & $95(72-100)$ & $22 / 1$ & $100(34-100)$ & $9 / 0$ \\
\hline Infección persistente a 6 meses por VPH $16 / 18$ & \multicolumn{2}{|c|}{ No fue objetivo del estudio } & $80(70-87)$ & $193 / 38$ \\
\hline Referencias & \multicolumn{2}{|c|}{34} & \multicolumn{2}{|c|}{33,35} \\
\hline
\end{tabular}

\footnotetext{
${ }^{\mathrm{c}}$ Estudios en animales no muestran evidencia de compromiso de la fertilidad o daño al feto.

d Por motivos éticos no es posible dejar a su evolución natural una NIE hasta el desarmollo de un CCU sin intervención terapéutica.

e Por motivos éticos no es factible realizar estudios moleculares de presencia de VPH en muestras de cuello uterino en niñas vírgenes.
} 
a protección contra tipos de VPH no contenidos en la vacuna:

Gardasil ${ }^{\circledR 3}$ (Brown D, et al. $47^{\text {th }}$ Interscience

Conference on Antimicrobial Agents and Chemotherapy (ICAAC), Chicago, E.U.A. 2007, Presentation \#G-1720b):

- Infección persistente a 6 meses VPH tipos 31 o 45: $45 \%$ (IC 95\%: 18-63)

- $\quad$ NIE 1 ó 3 por VPH tipos 31 o 45:

$45 \%$ (IC 95\%: 6-68)

- $\quad$ NIE 1 ó 3 por VPH tipos 31, 33, 45, 52 o 58:

$33 \%$ (IC 95\%: 8-52)

- NIE 2/3 por $10 \mathrm{VPH}$ oncogénicos

$38 \%$ (IC 95\%: 6-60)

Cervarix ${ }^{\circledR 33-35 \text { : }}$

- Infección incidente por VPH tipo 45:

94\% (IC 95\%: 63-100)

- Infección incidente por VPH tipo 31:

55\% (IC 95\%: 12-78)

- Infección persistente a 6 meses por VPH tipo 45: $60 \%$ (IC 95\%: 3-85)

- Infección persistente a 6 meses por VPH tipo 31: $36 \% \quad$ (IC 95\%: 0,5-60)

Estudios de costo-efectividad. Un estudio farmacoeconómico desarrollado por el Instituto Sabin para seis países de la Región de América Latina y el Caribe, incluido Chile, evaluó el impacto de una vacuna bivalente (VPH 16/18) (Constenla D, observaciones no publicadas). Los resultados de este estudio muestran que con una cobertura de vacunación de $70 \%$ se reduce en $39 \%$ el riesgo del CCU a lo largo de la vida en mujeres chilenas. Dicho de otra manera, la vacunación de $70 \%$ de una cohorte de mujeres de 12 años evitaría 2.767 casos de CCU a lo largo de la vida y 1.660 muertes.

A la luz de estos resultados en Chile, la vacunación de adolescentes contra VPH16/18 sería costo ahorrativa -esto es que el costo de la vacuna es menor que los costos médicos directos de las enfermedades prevenidas-, cuando se asume un costo por cada mujer vacunada de I $\$ 25^{f}$. La vacunación sería costo-efectiva -es decir, el costo para evitar la pérdida de un año de vida ajustado por discapacidad es menor a 3 veces el producto interno bruto (PIB) per capita- e incluso de mejor costo-efectividad que el tamizaje mediante Papanicolau si el costo por mujer vacunada es de I\$100.

En Chile, la vacunación de una cohorte de mujeres preadolescentes (12 años), considerando una cobertura de vacunación de $70 \%$, el costo de la vacunación de I\$360 por mujer y costos de prestaciones en los límites inferiores de nuestro país, significa un costo de más de I\$640 por año de vida salvado. Con base a los datos publicados, cuando el costo por mujer vacunada es de I\$360, las pruebas de tamizaje disponibles en Chile son de mayor costo-efectividad que la vacunación. Sin embargo, cuando el costo de la vacunación desciende, la estrategia más costo-efectiva es la vacunación de preadolescentes seguida de tres pruebas de tamizaje durante la vida.

Por otro lado, dos estudios farmacoeconómicos patrocinados por las compañías fabricantes de vacunas contra VPH y no publicados, encontraron que el uso de la vacuna en adolescentes chilenas sería costo-efectivo con un precio de vacuna no mayor de US\$100 por dosis.

\section{CONCLUSIONES}

Luego de una revisión de la epidemiología mundial y chilena, y de la información publicada 0 difundida en reuniones científicas respecto a las vacunas VPH disponibles, la que se ha resumido en este documento, el CCI concluye que:

- La infección por VPH y el CCU asociado mayoritariamente a los VPH 16 y 18 constituyen un importante problema de salud pública en nuestro país.

- Han sido notorios los avances logrados en Chile en las últimas décadas en diagnóstico, terapia y mortalidad del CCU. Sin embargo, preocupa

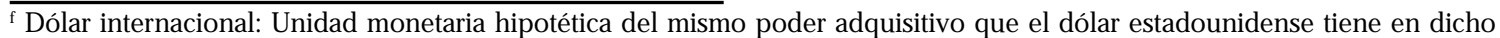
país en un momento dado. Se calcula utilizando la paridad del poder adquisitivo, o cantidad de unidades monetarias locales que se necesitan para adquirir, dentro de un determinado país, la misma cantidad de bienes que se comprarían en EEUU con un dólar estadounidense. Es calculado por el Banco Mundial y se usa para realizar comparaciones más válidas entre diferentes países y a lo largo del tiempo (por ejemplo comparaciones del producto bruto interno per capita).
} 
que aún ocurran más de 600 muertes anuales por esta causa y que la cobertura del sistema de tamizaje en Chile muestre un estancamiento desde hace varios años, existiendo 30\% de mujeres que no acceden a dicho sistema.

- No se dispone de información sistemática nacional que permita establecer la real carga de enfermedad debida a lesiones y cánceres de otras localizaciones en que participa el VPH ni de datos respecto a la importancia en salud pública de la infección por virus VPH 6 y 11. Sin embargo, se reconoce que las verrugas genitales constituyen un problema relevante tanto en carga de atención en los programas de infecciones de transmisión sexual, en el desmedro psicológico de los pacientes y en la complejidad y baja eficacia de las terapias.

- Las vacunas contra VPH recientemente licenciadas en Chile son seguras, bien toleradas e inmunogénicas.

- Es válido evaluar la eficacia de las vacunas como medidas de prevención del CCU mediante el uso de metas a evaluar sustitutas, como son las infecciones persistentes y NIE por VPH vacunales y los estudios puente como indicadores de eficacia en niñas.

- Ambas vacunas han demostrado una elevada eficacia para prevenir infecciones persistentes y NIE por los VPH contenidos en la vacuna, en la población de mujeres sin infección previa y que cumplen con el esquema de vacunación completo.

- La vacuna cuadrivalente ha demostrado una elevada eficacia para prevenir, además, lesiones intraepiteliales de cáncer de vulva y vagina en mujeres no infectadas y que completen el esquema de vacunación.

- Adicionalmente la vacuna cuadrivalente ha demostrado una elevada eficacia para prevenir la infección por los VPH 6 y 11 y vemugas genitales.

- Un análisis costo-efectividad realizado en forma independiente a las industrias farmacéuticas ha mostrado que la vacunación universal de adolescentes chilenas sería costo-efectiva para el país a un precio de I $\$ 100$ por mujer vacunada y costo- ahorrativa a un costo de I\$25 por mujer vacunada.

- El elevado costo actual de estos productos y su uso estrictamente privado generará una indeseada inequidad, pues beneficiará solamente a mujeres de estrato socioeconómico alto, quienes suelen tener adecuado y oportuno acceso al diagnóstico y terapia del CCU.

Por todo ello, el CCI formula las siguientes recomendaciones con respecto a la vacunación contra VPH en Chile:

- Vacunación de las adolescentes, idealmente antes del inicio de la actividad sexual, esto es, alrededor de los 12-13 años.

- Vacunación de adolescentes y mujeres, independiente de su edad, si no han iniciado actividad sexual.

- Acompañar la indicación de la vacuna con elementos educativos con relación a la sexualidad responsable, prevención de otras infecciones de transmisión sexual, eficacia de la vacuna y necesidad que las vacunadas permanezcan adheridas al sistema de tamizaje.

- Que la vacunación de las adolescentes y mujeres mayores que han iniciado actividad sexual se acompañe de adecuada información respecto a una posible menor eficacia de la vacuna en esta situación, considerando que ya podrían estar infectadas con VPH incluidos en las vacunas.

- Que las autoridades sanitarias consideren la incorporación de la vacunación universal contra VPH en el programa nacional de inmunizaciones.

En forma complementaria, el CCI recomienda:

- Fortalecer y apoyar las acciones de pesquisa y diagnóstico precoz abordadas por el Programa de Control del CCU. A su vez, incorporar indicadores de evaluación del seguimiento de las mujeres que presenten alteraciones del PAP.

- Implementar la vigilancia epidemiológica del VPH, lo que implica contar con un laboratorio de referencia capaz de confirmar la presencia del virus y los distintos genotipos en poblaciones definidas por el Programa de Control del CCU.

- La colaboración de los vacunatorios del sector privado que administren vacunas contra VPH, de modo que efectúen un registro sistemático de las personas vacunadas y las vacunas administradas, de forma de facilitar el seguimiento de esa población en caso que se incorpore la vacuna al Programa Nacional de Inmunización. 
Poten ciales conflictos de interés:

K. Abarca: ha recibido financiamiento de GSK para estudios clínicos de vacunas distintas a la vacuna VPH, ha asistido a congresos o reuniones científicas con auspicio de GSK y MSD, ha recibido honorarios de GSK y MSD como conferencista y por consultorías de expertos.

A. Muñoz: ha recibido financiamiento de GSK para estudios clínicos de vacunas distintas a la vacuna VPH, ha asistido a congresos o reuniones científicas con auspicio de GSK.

M. O'Ryan: ha recibido financiamiento de GSK por coordinar estudios clínicos de una vacuna distinta a la vacuna VPH; ha recibido honorarios de GSK como

\section{REFERENCIAS}

1. Parkin DM, Bray F. Chapter 2: The burden of VPHrelated cancers. In: Bosch FX, Cuzik J, Schiller JT, Garnett GP, Micheus A, Franco EL and Wright TC, editors. VPH vaccines and screening in the prevention of cervical cancer. Vaccine 2006; 24 (Suppl 3): S3/11-25.

2. Muñoz N, Castellsagué X, Berrington A, Gissmann L Chapter 1: VPH in the etiology of human cancer. In: Bosch FX, Cuzik J, Schiller JT, Garnett GP, Micheus A, Franco EL and Wright TC, editors. VPH vaccines and screening in the prevention of cervical cancer. Vaccine 2006; 24(Suppl 3): S3/1-10.

3. Cujford GM, Galuus S, Herrero R, Muñoz N, Snjders PJF, Vaccarelia S, and the IARC VPH Prevalence SuRveys Study Group. Worldwide distribution of human papillomavirus types in cytologically normal women in the International Agency for Research on Cancer VPH prevalence surveys: a pooled analysis. Lancet 2005; 366: 991-8.

4. Franceschi S, Herrero R, Clifford GM, Snijders PJF, ARsLan A, ANh PTH, aNd the IARC VPH Prevalence Surveys Study Group. Variations in the age-specific curves of human papillomavirus prevalence in women worldwide. Int J Cancer 2006; 119: 2677-84.

5. D'Souza GFA, Kreimer AR, Viscidi R, Pawlta M, Fakhry C, Koch WM et al. Case Control Study of Human Papillomavirus and Oropharyngeal Cancer. N Engl J Med 2007; 356: 1944-56.

6. Lacey CJN, Lowndes CM, Shah KV. Chapter 4: Burden and manegement of non-cancerous VPH-related conditions: VPH 6/11 disease. In: Bosch FX, Cuzik J, Schiller JT, Garnett GP, Micheus A, Franco EL and Wright TC, editors. VPH vaccines and screening in the prevention of cervical cancer. Vaccine 2006; 24(Suppl 3): S3/35-41.

7. Peñaloza-Placencia $M$, Montoya-Fuentes $H$, FloresMartínez SE, Fierro-Velasco FJ, Peñaloza-Gonzáaez JM, conferencista y por asesorías en una vacuna distinta a $\mathrm{VPH}$; se desempeña como "Chairman" de un grupo técnico consultor de MSD de una vacuna distinta a VPH. Ema Ripoll: ha asistido a congresos o reuniones científicas con auspicio de GSK.

M. Teresa Valenzuela: ha asistido a dos reuniones científicas, no relacionadas con la vacuna VPH, con auspicio de GSK y MSD.

R. Vergara: ha recibido financiamiento de GSK para estudios clínicos de vacunas distintas a la vacuna VPH, ha asistido a congresos o reuniones científicas con auspicio de GSK Vivian Luchsinger y Jorge Jiménez no declaran conflictos de interés.

Sánchez-Corona J. Molecular Identification of $7 \mathrm{Hu}-$ man Papillomavirus types in Recurrent Respiratory Papillomatosis. Arch Otoralyngol Head Neck Surg 2000; 126: 1119-23.

8. Burchell AN, Winer RL, De Sanjosé S, Franco EL Chapter 6. Epidemiology and transmission dynamics of genital VPH infection. In: Bosch FX, Cuzik J, Schiller JT, Garnett GP, Micheus A, Franco EL and Wright TC, editors. VPH vaccines and screening in the prevention of cervical cancer. Vaccine 2006; 24(Suppl 3): S3/52-62.

9. Moscicki AB, Achiffman M, KJaer S, Viшa LL Chapter 5: Updating the natural history of HPV and anogenital cancer. In: Bosch FX, Cuzik J, Schiller JT, Garnett GP, Micheus A, Franco EL and Wright TC, editors. VPH vaccines and screening in the prevention of cervical cancer. Vaccine 2006; 24(Suppl 3): S3/4251.

10. Departamento de Estadísticas e Información en Salud - Unidad de Cáncer Ministerio de Salud, 2004.

11. CITOEXPERT/Departamento de Estadísticas e Información en Salud (DEIS)-Unidad de Cáncer, Ministerio de Salud, 2005.

12. Donoso E, Cuelo M, Vilarroel L Reducción en la mortalidad por cáncer cérvico uterino en Chile, 1990-2003. Rev Chil Obstet Ginecol 2006; 71: 307-12.

13. Ferreccio C, Prado R, Luzoro A, Ampuero S, Snijders P, MejJer $C$ et al. Population-Based Prevalence and Age Distribution of Human Papillomavirus Among Women in Santiago, Chile. Cancer Epidemiol Biomarkers Prev 2004; 13: 2271-6.

14. Ferreccio C, Corvalán A, Margozzin P, Viviani P, Gonzáigz C, Aguilera $\mathrm{X}$ Et al. Baseline assessment of prevalence and geographical distribution of HPV types in Chile using self-collected vaginal samples. BMC Public Health 2008; 8: 78 doi: 10.1186/1471-2458-8-78.

15. República de Chile, Ministerio de Salud. Encuesta Nacional de Salud 2003. Resumen ejecutivo. El Vigía (Chile) 2004; 8: 1-20. 
16. Melo A, Montenegro S, Hooper T, Capurro I, Roa J, RoA I. Tipificación del virus papiloma humano (VPH) en lesiones preneoplásicas y carcinoma del cuello uterino en mujeres de la IX región- Chile. Rev Méd Chile 2003; 131: 1382-90.

17. CONASIDA, Ministerio de Salud. Enfermedades de Transmisión Sexual, Chile. Boletín № 2; páginas 6-7.

18. CONASIDA, Ministerio de Salud. Enfermedades de Transmisión Sexual, Chile. Boletín № 5; páginas 9-10.

19. Valenzuela MT, Pereira A, Cavada G, Pantoja J, FANJELREN V. Implementación de un sistema de monitoreo y evaluación en el entorno clínico del cambio de comportamiento de los consultantes de ETS. Informe Final Proyecto Fondo Global, 2007.

20. Instituto Nacional de la Juventud. Cuarta Encuesta Nacional de Juventud. Resultados Generales. 2003

21. Ministerio de Salud de Chile, OMS, OPS, CDC. Encuesta Mundial de Salud Escolar. Chile 2004.

22. Laboratorio Merck. Gardasil ${ }^{\circledR}$. Folleto de información al profesional.

23. Giannini SL, Hanona E, Moris P, Van Mechelen M, Morel S, Dessy F ET AL. Enhanced humoral and memory B cellular immunity using VPH 16/18 L1 VLP vaccine formulated with the MPL/alumimium SALT combination (AS04) compared with aluminium SALT only. Vaccine 2006; 24: 5937-49.

24. Laboratorio GSK. Cervarix ${ }^{\circledR}$. Folleto de información al profesional.

25. Vima LL, Ault KA, Giulano AR, Costa RL, Petta CA, ANDRADE RP ET AL. Immunologic responses following administration of a vaccine targeting human papillomavirus types 6, 11, 16 and 18. Vaccine 2006; 24: 5571-83.

26. Harper DM, Franco EL, Wheeler C, Ferris DG, Jenkins D, SCHUIND A ET AL Efficacy of a bivalent L1 vinus-like particle vaccine in prevention of infection with human papillomavinus types 16 and 18 in young women: a randomized controlled trial. Lancet 2004; 364: 1757-65.

27. Block SL, Nolan T, Sattler C, Barr E, Giacoletti KED, MARCHANT CD ET AL Companison of the immunogenicity and reactogenicity of a prophylactic quadrivalent human papillomavinus (types 6, 11, 16 and 18) L1 virus-like particle vaccine in male and female adolescents and young adult women. Pediatrics 2006; 118: 2135-45.

28. Reisinger KS, Block SL, Lazcano-Ponce E, Samakoses R, Esser MT, ERICK J ET AL. Safety and Persistent Immunogenicity of a Quadrivalent Human Papillomavirus Types 6, 11, 16, 18 L1 Virus-Like Particle Vaccine in Preadolescents and Adolescents: A Randomized Controlled Trial. Pediatr Infect Dis J 2007; 26: 201-9.

29. Pedersen C, Petaja T, Strauss G, Rumke HC, Poder A, RichARDUS JH ET AL. Immunization of early adolescent females with human papillomavirus type 16 and 18 L1 virus-like particle vaccine containing AS04 adjuvant. J Adolesc Health 2007; 40: 564-71.

30. Pérez G, Lazcano-Ponce E, Hernández-Avila M, García PJ, MuÑoz N, Viшa L ET aL. Safety, immunogenicity, and efficacy of quadrivalent human papillomavirus (types $6,11,16,18)$ L1 virus-like-particle vaccine in Latin American women. Int J Cáncer 2008; 122: 1311-18.

31. Vima LL, Costa RLR, Petta CA, Andrade RP, Pavoneen J, IVERSEN OE. High sustained efficacy of a prophylactic quadrivalent human papillomavinus types 6/11/16/18 L1 virus-like particle vaccine through 5 years of follow-up. Br J Cancer 2006; 95: 1495-66.

32. Olsson SE, Viua LL, Costa RLR, Petta CA, Andrade RP, MALMF C ET AL. Induction of immune memory following administration of a prophylactic quadrivalent human papillomavirus (HPV) types 6/11/16/18 L1 virus-like particle (VLP) vaccine. Vaccine 2007; 25: 4931-9.

33. Harper DM, Franco EL, Wheeler C, Moscicki AB, RomanowsKi B, Rotel-MarTins CM ET AL. Sustained efficacy up to 4,5 years of a bivalent L1 virus-like particle vaccine against human papillomavirus types 16 and 18: follow-up from a randomized control trial. Lancet 2006; 367: 1247-55.

34. Food and Drug Administration. Product approval information-licensing action. Gardasil (quadrivalent human papillomavirus types $6,11,16,18)$. Merck \& Co, Whitehouse Station, NJ: Disponible en: http:// www.fda.gov/cber/products/gardasil.htm, accedido 11 sept 2008.

35. Paavonen J, Jenkins D, Bosch FX, Naud P, Salmerón J, WheELER CM ET AL. Efficacy of a prophylactic adjuvanted bivalent L1 virus - like- particle against infection with human papillomavirus types 16 and 18 in young women: an interim analysis of a phase III double blind, randomised controlled trial. Lancet 2007; 369: 2161-70.

36. Garland SM, Hernández-Avila M, Wheeler CM, Pérez G, HARPER DM, LEODOLTER S ET AL. Quadrivalent Vaccine against Human Papillomavirus to Prevent Anogenitals Lesions. N Engl J Med 2007; 356: 1928-43.

37. The Future II Study Group. Quadrivalent Vaccine against Human Papillomavirus to Prevent High-Grade Cervical Lesions. N Engl J Med 2007; 356: 1915-27.

38. The Future II Study Group. Effect of prophylactic human papillomavinus L1 vinus-like-particle vaccine on risk of cervical intraepithelial neoplasia grade 2, grade 3 and adenocarcinoma in situ: a combined analysis of four randomized clinical trials. Lancet 2007; 369: 1861-8.

39. Vilia LL, Costa RL, Petta CA, Andrade RP, Ault KA, Giulano AR ET AL. Prophylactic quadrivalent human papillomavirus (types 6, 11, 16, 18) L1 virus-like particle vaccine in young women: a randomized double blind placebo controlled multicentre phase II efficacy trial. Lancet Oncol 2005; 6: 271-8.

40. Hildesheim A, Herrero R, Wacholder S, Rodríguez AC, Solomon D, Bratti MC ET AL. Effect of Human Papillomavirus 16/18 L1 Viruslike Particle Vaccine Among Young Women With Preexisting Infection. A Randomized Trial. JAMA 2007; 208: 743-53. 